\title{
COMO DIFERENTES TEORES DE BIODIESEL NO DIESEL AFETAM AS EMISSÕES DE ALDEÍDOS EM MOTORES E VEÍCULOS
}

\author{
Tadeu Cavalcante Cordeiro de Melo ${ }^{1}$, José Roberto de Souza Rocha ${ }^{1}$, Renato de Arruda \\ Penteado Neto $^{2}$, Rodrigo Soares Ferreira ${ }^{2}$ \\ ${ }^{1}$ PETROBRAS - CENPES \\ ${ }^{2}$ LACTEC
}

E-mails: tcm@petrobras.com.br, jrrocha@petrobras.com.br, renato@lactec.org.br, rodrigo.ferreira@lactec.org.br

\section{RESUMO}

Em 2008, o CONAMA (Conselho Nacional do Meio Ambiente) publicou a resolução 408/08 estabelecendo que o IBAMA (Instituto Brasileiro do Meio Ambiente e dos Recursos Naturais Renováveis) seria responsável por fornecer um procedimento de medição de aldeídos em motores diesel. Com base nesse procedimento as montadoras de veículos deveriam reportar valores típicos de emissões de aldeídos até dezembro de 2012. Em 2009, a AEA (Associação de Engenharia Automotiva) criou um grupo de trabalho para discutir e propor um método de medição de emissões de aldeídos em veículos e motores diesel.

Desde 2008, alguns institutos de pesquisa estudam metodologias de medição das emissões de aldeídos em veículos e motores diesel com diferentes tipos de biodiesel. Esse artigo irá apresentar uma revisão bibliográfica sobre a formação de aldeídos e metodologias de medição das emissões destes compostos com o uso da técnica analítica de cromatografia líquida de alta eficiência (HPLC) e por espectroscopia de infravermelho (FTIR - Fast Fourier Transform Infrared analyzer). Também serão apresentados resultados de emissões com até $20 \%$ de adição de biodiesel usando dois tipos de oleaginosas em diferentes veículos e em um motor diesel EURO III.

\section{INTRODUÇÃO}

No Brasil, o PROCONVE (Programa de Controle da Poluição do Ar por Veículos Automotores) definiu em 1992 limites de emissões para os aldeídos totais (formaldeído mais acetaldeído) apenas para veículos a gasolina e a etanol. Em 2005, foi publicado pela CETESB (Companhia de Tecnologia de Saneamento Ambiental) um artigo na AEA relatando valores significativos de emissões de aldeídos para alguns modelos de picapes e SUVs (Sport Utility Vehicle) antigos. Os ensaios foram feitos em dinamômetro de chassis segundo a NBR 6601 (emissões gasosas e ciclo de condução) e a NBR 12026 (método DNPH- 2,4 Dinitrofenilhidrazina e cromatografia HPLC) para análise de emissões de aldeídos [1, 2, 3].

Em 2008, o CENPES (Centro de Pesquisas e Desenvolvimento da PETROBRAS) junto com 
o LACTEC (Instituto de Tecnologia para o Desenvolvimento), decidiram estudar metodologias para medição de aldeídos em veículos e motores diesel usando as técnicas de HPLC (High Performance Liquid Chromatography) e FTIR (Fourier Transform Infra-Red Analyzer).

Em 2008, o CONAMA publicou a legislação 403/08 com os novos limites CONAMA P7, e estabeleceu que o IBAMA deveria fornecer um procedimento para a medição de aldeídos em veículos diesel até o mês de dezembro de 2010. Também incluído na legislação, estava a necessidade das montadoras informarem os valores típicos de emissões de aldeídos de seus veículos e motores até dezembro de 2012.

Como não existia procedimento internacional para essa medição, foi criado em 2009 um grupo técnico na AEA para estudar o assunto e subsidiar o IBAMA na elaboração de uma metodologia para a medição de aldeídos totais em motores e veículos do ciclo diesel.

Esse artigo aborda as metodologias desenvolvidas pelo CENPES e LACTEC para a medição de aldeídos em veículos do ciclo diesel e também como a adição de biodiesel pode afetar as emissões desse poluente.

\section{PROPRIEDADES DOS ALDEÍDOS E LEGISLAÇÃO}

O grupo aldeído também é conhecido como álcool deshidrogenado contendo uma parte com hidrocarbonetos e uma com o grupo carbonila- CHO. Eles são formados na câmara de combustão quando o processo de reação de pré-combustão se inicia.

Eles se formam principalmente em baixas temperaturas, sendo assim, a concentração máxima desses poluentes ocorrerá na partida a frio do motor e em situações de baixa carga.

A figura 1 mostra o grupo funcional do aldeído com fórmulas estruturais do formaldeído e do acetaldeído, enquanto a figura 2 mostra a reação de formação do acetaldeído a partir da combustão do etanol.

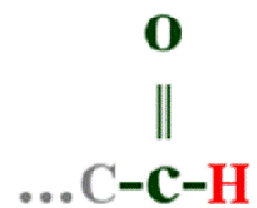

(a)<smiles>C=O</smiles>

(b)<smiles>CC=O</smiles>

(c)

Figura 1. Grupos Funcionais de (a) Aldeído, (b) Formaldeído, (c) Acetaldeído.<smiles>CCCC(=O)OC(C)C(C=O)C=O</smiles>

Figura 2. Formação de aldeídos a partir da oxidação do etanol. 
Os aldeídos são precursores de ozônio e alguns deles são considerados carcinogênicos. Os veículos movidos a etanol emitem cerca de $90 \%$ de acetaldeído e $10 \%$ de formaldeído, enquanto que no motor diesel a formação é $70 \%$ de formaldeído e $30 \%$ de acetaldeído.

No Brasil, desde 1992, existe uma legislação para os aldeídos totais (somatório das concentrações de formaldeído e acetaldeído) apenas para veículos a gasolina, Flex e etanol. Os ensaios são conduzidos em dinamômetro de chassis segundo a NBR 6601 (emissões de gases e condução do ciclo) e NBR 12026 (medição de aldeídos por cromatografia líquida método DNPH - 2,4 dinitrofenilhridrazina) [1,3].

A amostra de escapamento do veículo após ser diluída de forma controlada, passa um fluxo de $2 \mathrm{~L} / \mathrm{min}$ através de impingers (tubo de vidro especial contendo solução de acetonitrila e 2,4 DNPH). Desta forma, os aldeídos presentes na amostra gasosa reagem com o 2,4-DNPH, sendo suas concentrações posteriormente determinadas por meio da análise da solução por HPLC. Na sequência é realizado o cálculo de conversão de concentração de miligramas por litro $(\mathrm{mg} / \mathrm{L})$, para concentração em massa $(\mathrm{g})$ por quilômetro $(\mathrm{km})$ percorrido.

Desde 2009, o limite de emissões de aldeídos para veículos do ciclo Otto de passageiros é de $0,02 \mathrm{~g} / \mathrm{km}$ (PROCONVE L5). Para veículos comerciais leves (SUVs ou picapes), movidas a gasolina ou etanol, o limite é de $0,06 \mathrm{~g} / \mathrm{km}$ para a fase L3 (1997) e de 0,04 g/km para a fase L5 (2009).

Com relação aos veículos a diesel, como mencionado anteriormente, não há legislação e procedimento internacional padronizado para a medição de aldeídos.

\section{REVISÃO BIBLIOGRÁFICA}

Em 2005, a CETESB realizou diversos ensaios de emissões em veículos a diesel para medir as emissões de aldeídos [2]. Foram testados, segundo a Norma Brasileira NBR 6601, quatro veículos diesel, de elevada quilometragem, no laboratório da CETESB, em São Paulo. São apresentadas as técnicas utilizadas para a coleta das amostras e um diagrama de teste dos veículos. O artigo cita que, quando comparados com as emissões reais dos veículos leves equipados com motores de ignição por centelha, fabricados em 1995, de maneira geral, os veículos diesel emitem mais aldeídos e a magnitude se assemelha aos veículos a álcool.

$\mathrm{O}$ artigo chama a atenção para o fato de que a restrição na legislação para aldeídos nos veículos a diesel não é procedente, visto que os resultados desse estudo demonstraram que os veículos movidos a diesel da categoria testada emitiram muito mais aldeídos que os veículos do ciclo Otto. O estudo ainda destaca o fato de que o estabelecimento de limites para as emissões de aldeídos dos veículos de ignição por centelha se mostrou eficiente na redução dos níveis emitidos, principalmente com a utilização de conversores catalíticos.

Esses resultados mostraram ainda que a emissão de acetaldeído em veículos diesel variou de 5,9 a $45,4 \mathrm{mg} / \mathrm{km}$ e a de formaldeído variou de 16,5 a $115,2 \mathrm{mg} / \mathrm{km}$. A emissão média para a soma dos aldeídos foi de $58,7 \mathrm{mg} / \mathrm{km}$, variando de 22,5 a $160 \mathrm{mg} / \mathrm{km}$. A proporção entre os dois aldeídos se manteve constante, e próxima de $74 \%$ de formaldeído e $26 \%$ de acetaldeído.

Os autores sugerem a realização de estudos com amostras maiores, e ensaios em 
dinamômetros de motores para determinação dos aldeídos, determinação do fator de deterioração destes veículos e criação de inventário das emissões de poluentes. Concluem que a emissão de aldeídos é significativa e importante, sendo necessário o estabelecimento de limite para veículos diesel.

Apesar da emissão de aldeídos não ser regulamentada no Brasil e em outros países, existem diversas pesquisas sendo feitas a nível internacional. De forma a se conhecer mais sobre as diferentes metodologias de medição usadas, foi feita uma extensa revisão bibliográfica, sendo os principais artigos destacados a seguir.

Caplain et al. [4] mediram gases de escape diluído de veículos diesel usando cartuchos com $\mathrm{DNPH}$, e verificaram que o formaldeído era o principal tipo de aldeído presente. Shiotani et al. [5] mediram aldeídos (formaldeído, acetaldeído, acroleína e benzaldeído) de motores "off-road" em banco de provas. As medidas foram feitas usando um cartucho de sílica gel impregnado com 2,4 DNPH com amostragem diluída por mini-túnel usando uma bomba de vazão $400 \mathrm{~mL} / \mathrm{min}$ durante 10 minutos. A amostra foi depois analisada por HPLC. Os autores também analisaram a amostra bruta (formaldeído e acetaldeído) usando um analisador HORIBA FTIR modelo 4000 FT. Os aldeídos apresentaram valores mais altos para baixas cargas (10\% de torque máximo) e boa uma correlação foi encontrada entre as medições com FTIR e HPLC para o formaldeído $(\mathrm{R} 2=0,9)$, mas não para o acetaldeído.

Arapaki et al. [6] usaram um cartucho com 2,4 DNPH, com uma vazão de gás de amostragem de $150 \mathrm{~mL} / \mathrm{min}$, com análise por HPLC. Peng et al. [7] usaram cartucho DNPH S10, Supelco, $1 \mathrm{~L} / \mathrm{min}$ de vazão durante 20 minutos. Também um sistema HPLC foi usado para analisar 14 diferentes aldeídos. Pi-Qiang et al. [8]usaram um sistema AVL FTIR para a medição de amostra bruta da exaustão de um motor diesel instalado em banco de provas. Karavalakis et al. [9] coletaram amostras diluídas com um saco de tedlar e com um cartucho chomafix-DNPH, foi usada uma vazão de $150 \mathrm{~mL}$ para a amostragem, totalizando $3 \mathrm{~L}$ no total. Foi usado um sistema HPLC nessa análise. Guarieiro et al. [10] usaram um sistema do tipo impinger com 2,4 DNPH e HPLC para analisar até 18 diferentes compostos carbonílicos diferentes de carbonila de um motor diesel pequeno de 2 cilindros.

Shah et al. [11] e He et al [12] usaram um sistema de mini-diluição com razão de diluição em torno de 8 . Foi feito o bombeamento da amostra através de um cartucho com uma vazão de $260 \mathrm{~mL} / \mathrm{min}$. durante 30 minutos. Foi utilizada a norma ISO 8178 e ensaiado um motor diesel de 4 cilindros. Storey et al.[13] usaram um sistema de diluição similar ao de Shah et al., mas a amostra foi coletada apenas na marcha lenta do motor com uma vazão de $1 \mathrm{~L} / \mathrm{min}$ durante 90 minutos.

Em 2011, Karavalakis et al. [14] investigaram o efeito de misturas de biodiesel nas emissões regulamentadas e não regulamentadas de um veículo de passageiro equipado com um motor Euro IV, catalisador de oxidação para diesel e DPF (Diesel Particle Filter). Os testes foram realizados nos ciclos New European Driving Cycle (NEDC) e Artemis. Foram utilizadas misturas com biodiesel de soja e óleo usado de fritura, adicionados a diesel de baixo teor de enxofre nas proporções de 20, 30, e 50\%. Os resultados mostraram que o uso de biodiesel ocasionou numa redução do $\mathrm{CO}$ e do HC. No entanto um notável aumento no NOx foi observado proporcionalmente à participação do biodiesel nas misturas, assim como as emissões de $\mathrm{CO}_{2}$ e o consumo de combustível. A influência do tipo e das características dos 
combustíveis foi particularmente notada nas emissões não legisladas. O uso de óleo oxidado de fritura levou a um aumento significativo no formaldeído, acetaldeído e acroleína.

Em 2012, Anderson [15] publicou um estudo realizado em veículos leves do ciclo diesel, abastecidos com misturas de óleo diesel e biodiesel nas proporções de B5 até B100. Foi usado biodiesel de soja, canola, palma, de origem animal e de óleo usado de cozinha. Os testes realizados em dinamômetro de chassis mostram que a emissão de hidrocarbonetos não teve uma variação estatisticamente significativa quando foram utilizadas as misturas com o biodiesel. Para o NOx, as misturas de biodiesel resultaram em aumento estatisticamente significativo para B10, B20, B30, B50 e B100. As emissões de CO reduziram apenas quando utilizada a mistura B20, e as demais misturas não levaram a alterações de significância estatística. $\mathrm{O} \mathrm{CO}_{2}$ apenas apresentou uma leve redução em $\mathrm{B} 10$. O efeito do biodiesel no material particulado provocou uma redução para B10, B20, B30 e B50. No que diz respeito aos aldeídos, foram analisadas as misturas B10, B20 e B30. Anderson mostra que a presença do biodiesel na mistura resultou num aumento da ordem de 25 a $35 \%$ no formaldeído para B10, B20 e B30. Os resultados para o acetaldeído comprovam um aumento na mistura B30, e sugerem uma tendência de aumento nas misturas B10 e B20.

Um estudo realizado em 2012 por Chin et al.[16], na Universidade de Michigan, avaliou as emissões geradas por dois motores, de 1,7 e 6,4 litros, em condição de carga e marcha lenta. Os motores foram alimentados por biodiesel B20 e diesel de baixo teor de enxofre (ULSD). Para o motor de 1,7 litros, operando em regime de alta carga, com B20 e equipado com um catalisador de oxidação, foi observada uma elevação no NMHC, NOx, formaldeído e compostos orgânicos voláteis (COV), se comparado com o diesel ULSD. Para o motor de 6,4 litros, sob carga e com B20, houve uma redução no MP, HC, formaldeído, COVs. Foi observado, no entanto, um aumento do NOx. Os autores concluem o trabalho afirmando que, apesar do uso do B20 reduzir a emissão de muitos poluentes, os teores de formaldeído e o benzeno aumentaram no motor de 1,7 1. Apesar dos poluentes não legislados representarem uma fração pequena do total de emissões, estes devem ser considerados devido à importância de sua toxicidade.

Pode ser visto da literatura que a maioria dos artigos reporta o uso de da técnica de medição de cromatografia líquida HPLC com cartucho de sílica impregnado com 2,4 DNPH e utilizando uma amostra diluída. Esse procedimento foi adotado para uso nos ensaios experimentais desse trabalho.

\section{COMBUSTÍVEIS}

Foi utilizado um diesel de $50 \mathrm{ppm}$ de enxofre (S50), além de biodiesel de sebo e soja adquiridos de fornecedores do mercado para preparo de misturas de biodiesel nos teores de $5 \%$ (B5) e 20\% (B20). Os resultados das principais análises feitas pelo CENPES para o diesel S50 e suas misturas com o biodiesel são apresentados na Tabela 1. 
Tabela 1. Principais análises dos combustíveis usados no trabalho.

\begin{tabular}{|c|c|c|c|}
\hline Amostra & $\begin{array}{c}\text { Massa especifica a } 20^{\circ} \mathrm{C}, \mathrm{kg} / \mathrm{m}^{3} \\
\text { ASTM D } 4052\end{array}$ & $\begin{array}{l}\text { Viscosidade a } 40^{\circ} \mathrm{C}, \mathrm{mm}^{2} / \mathrm{s} \\
\text { ASTM D } 445\end{array}$ & $\begin{array}{l}\text { Número de cetano derivado } \\
\text { ASTM D6890 }\end{array}$ \\
\hline S50 & 837,8 & 2,878 & 50,4 \\
\hline B100 de soja & 881,3 & 4,125 & \\
\hline B5 soja & 839,8 & 2,918 & 48,3 \\
\hline B20 soja & 846,0 & 3,053 & 48,0 \\
\hline B100 de sebo & 873,6 & 4,637 & \\
\hline B5 sebo & 839,4 & 2,936 & 50,8 \\
\hline B20 sebo & 844,6 & 3,139 & 53,1 \\
\hline
\end{tabular}

\section{VEÍCULOS E MOTORES}

\subsection{VEÍCULOS}

Foram usados três veículos diesel das fases do PROCONVE L-3: V1 (picape), 2005, V2 (SUV), 2005, mod. 2006 e V3 (SUV) 2004, modelo 2005, com catalisador de oxidação (CO e THC), sem sonda lambda, com EGR e com SCV (Swirl Control Valve).

As principais características dos motores dos veículos utilizados estão apresentadas da Tabela 2.

Tabela 2. Principais características dos motores dos veículos utilizados.

\begin{tabular}{cc|c|c}
\hline Característica & V1 & V2 & V3 \\
\hline Tipo de Injeção & Direta & Direta & Direta \\
\hline $\begin{array}{c}\text { Velas de Pré- } \\
\text { aquecimento }\end{array}$ & Não & Sim & Sim \\
\hline $\begin{array}{c}\text { Sistema de Partida a } \\
\text { Frio }\end{array}$ & Mecânico & Eletrônico & Eletrônico \\
\hline $\begin{array}{c}\text { Turbo Compressor } \\
\text { Sistema de Injeção }\end{array}$ & $\begin{array}{c}\text { Bomba } \\
\text { Distribuidora }\end{array}$ & Common Rail & $\begin{array}{c}\text { Bomba } \\
\text { Distribuidora }\end{array}$ \\
\hline Controle da Injeção & Mecânico & Eletrônico & Eletrônico \\
\hline
\end{tabular}




\subsection{MOTORES}

Para as análises de aldeídos foi usado um motor EURO III, de propriedade do CENPES, com as principais características listadas a seguir:

- Número e disposição dos cilindros : 4 cilindros em linha;

- Cilindrada total : 4,250 litros ;

- Taxa de compressão: 18:1;

- Rotação de marcha-lenta : 900 rpm;

- Potência máxima : $130 \mathrm{~kW}$;

- Velocidade de potência máxima : 2500 rpm;

- Torque máximo : $680 \mathrm{Nm}$;

- Sistema de injeção de combustível: "Unit Pump System" ;

- Alimentação de ar para os cilindros por turbocompressor ;

- Classificação da tecnologia de controle de emissões: EURO III.

\section{METODOLOGIAS}

\subsection{MEDIÇÂO DE ALDEÍDOS EM VEÍCULOS LEVES}

Para a amostragem da exaustão foi usado um sistema da empresa Horiba com o uso de sacos de diluição e análise dos gases por bancada Horiba Mexa 7000. O escapamento diluído foi controlado por um sistema do tipo impinger com vazão constante de $2 \mathrm{~L} / \mathrm{min}$. Cada par de impinger continha cerca de $50 \mathrm{~mL}$ de acetonitrila e 2,4-DNPH misturado com ácido perclórico.

Um cromatógrafo líquido de alta eficiência (HPLC) foi usado para as análises dos aldeídos. Em paralelo, foi estudada a presença de aldeídos no filtro de material particulado usando a técnica cromatográfica . Não foi constatada presença significativa de aldeídos no filtro de particulados. Algumas modificações foram feitas na norma NBR 12026 de medição de aldeídos. [2]:

- Comprimento de Onda - $365 \mathrm{~nm}$ e coluna - fase reversa C18 com fase móvel acetronitrila: água - 70\%:30\% com volume de injeção de $10 \mu \mathrm{L}$.

- Tempo de análise em torno de 10 minutos para o formaldeído e acetaldeído e 15 minutos mais para os outros aldeídos. Vazão no impinger - $2 \mathrm{~L} / \mathrm{min}$.

A figura 3 mostra o sistema de amostragem com um mini-túnel de diluição e um filtro de material particulado (MPF). 


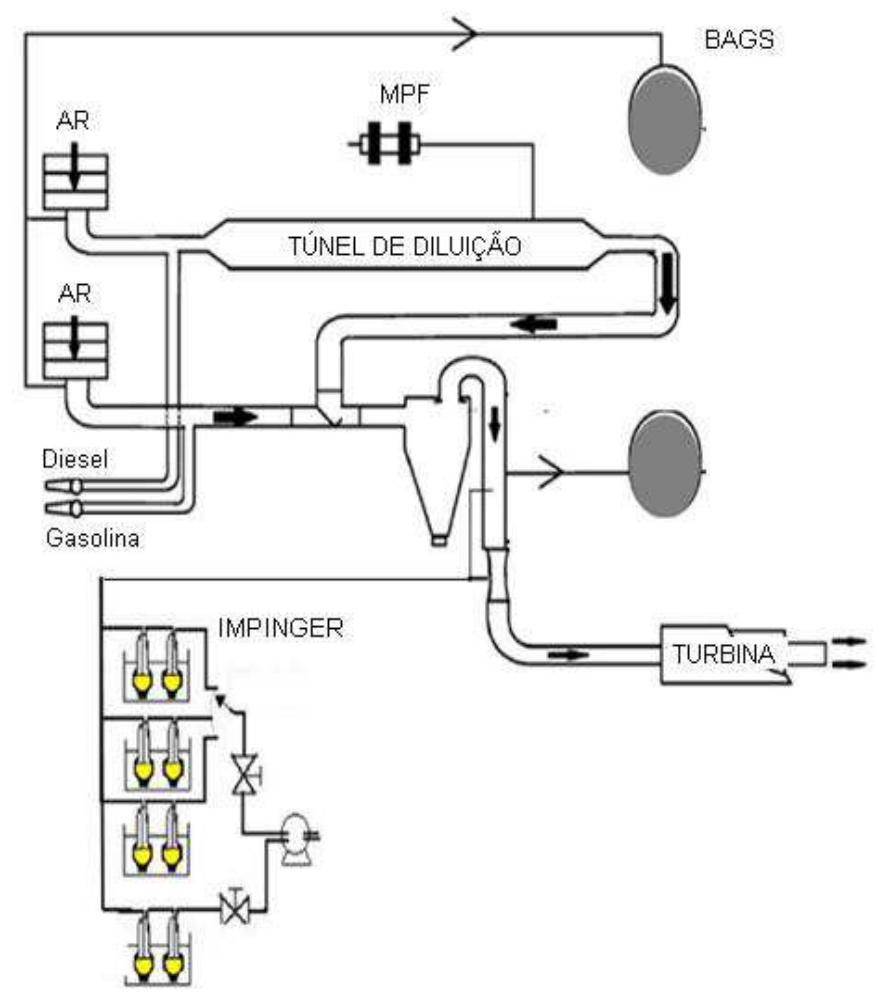

Figura 3. Sistema de amostragem de aldeídos para veículos leves a diesel.

Dentre os vários componentes eventualmente presentes nos gases de exaustão e capturados pela solução absorvedora de 2,4-DNPH (método NBR 12026), foram destacados os seguintes: Formaldeído, Acetaldeído, Acroleína, Acetona, Propionaldeído, Crotonaldeído, Metacroleína, 2-Butanona, Butiraldeído, Benzaldeído, Valeraldeído, m-Tolualdeído e Hexaldeído. A presença destes compostos foi avaliada por meio da comparação com padrões cromatográficos (mix de derivados de compostos carbonílicos) certificados injetados nas mesmas condições no cromatógrafo e em diferentes concentrações. Na figura 4 é mostrado um cromatograma do padrão utilizado destacando os derivados carbonílicos presentes no mix.

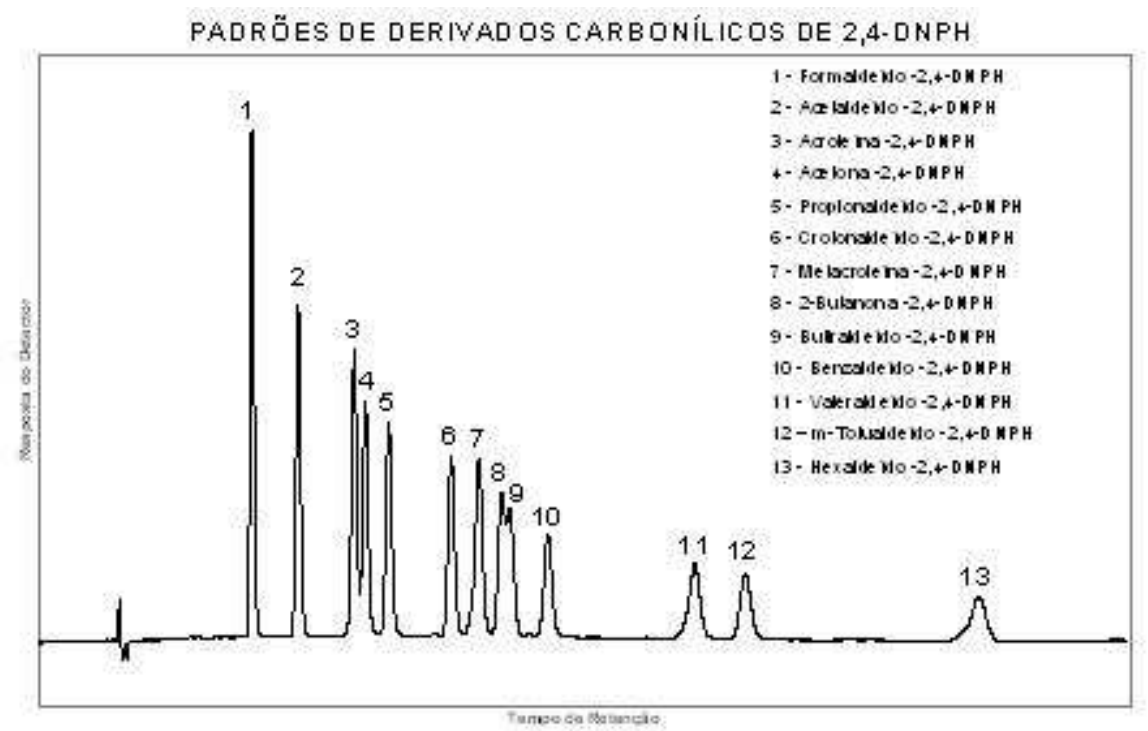

Figura 4. Cromatograma dos padrões de derivados carbonílicos. 
Foi utilizado também o equipamento AVL SESAM FTIR (Fourier Transform Infrared Spectroscopy) para os estudos de formação de aldeídos ao longo do tempo. Esse equipamento é capaz de analisar simultaneamente até 23 poluentes incluindo diferentes tipos de aldeídos.

\subsection{MEDIÇÂO DE ALDEÍDOS EM MOTOR}

Para a realização do ensaio de emissões de aldeídos em motor EURO III (CONAMA P5) foi usado o ciclo de motor ESC (European Stationary Cycle) segundo o procedimento da norma brasileira ABNT NBR 6601 e foram feitas pelo menos 3 repetições com cada combustível para possibilitar um tratamento estatístico.

Para a análise de aldeídos, inicialmente foi usada a técnica de FTIR, pois era mais simples de se fazer a coleta de amostra do escapamento do motor de forma bruta. $\mathrm{O}$ equipamento FTIR fornece a concentração em ppm dos diferentes aldeídos, sendo que para o cálculo da massa em $\mathrm{g} / \mathrm{kWh}$ foi usado o procedimento da norma NBR 15634 aplicando as densidades dos aldeídos da, norma NBR 12026.

Foi feita uma correlação da bancada FTIR com a bancada de emissões gasosas, que mede os poluentes legislados e a correlação foi satisfatória (SIMEA DAEMME,2012) . Com relação aos aldeídos, os valores encontrados com a análise de FTIR foram bem mais elevados do que com a técnica de cromatografia, dessa forma o FTIR foi usado apenas para verificação da tendência de aumento ou redução das emissões de aldeídos em função do teor de biodiesel.

O método escolhido para a quantificação de aldeídos desse trabalho foi a cromatografia líquida com amostragem por cartucho impregnado com DNPH (DiNitro fenil hidrazina) segundo recomendação de MELO et al. (SAE 2009).

Como o cartucho de DNPH pode sofrer uma saturação devido ao excesso de NOx, presente no gás de escapamento de um motor diesel, não foi possível a amostragem do escapamento de forma bruta. Para se ter uma amostra diluída do escapamento, a forma encontrada foi se fazer uma modificação no equipamento de amostragem de material particulado (Smart Sampler), de forma a se incluir uma bomba de amostragem para impregnação do cartucho, sem que a mesma interferisse na medição de material particulado. A vantagem desse sistema foi permitir o cálculo do valor de emissão dos aldeídos em $\mathrm{g} / \mathrm{kWh}$ com o uso dos mesmos fatores de diluição empregados no cálculo da emissão de material particulado.

O esquema com modificações feitas no equipamento de amostragem de material particulado para a amostragem de aldeídos é exemplificado na Figura 5. 


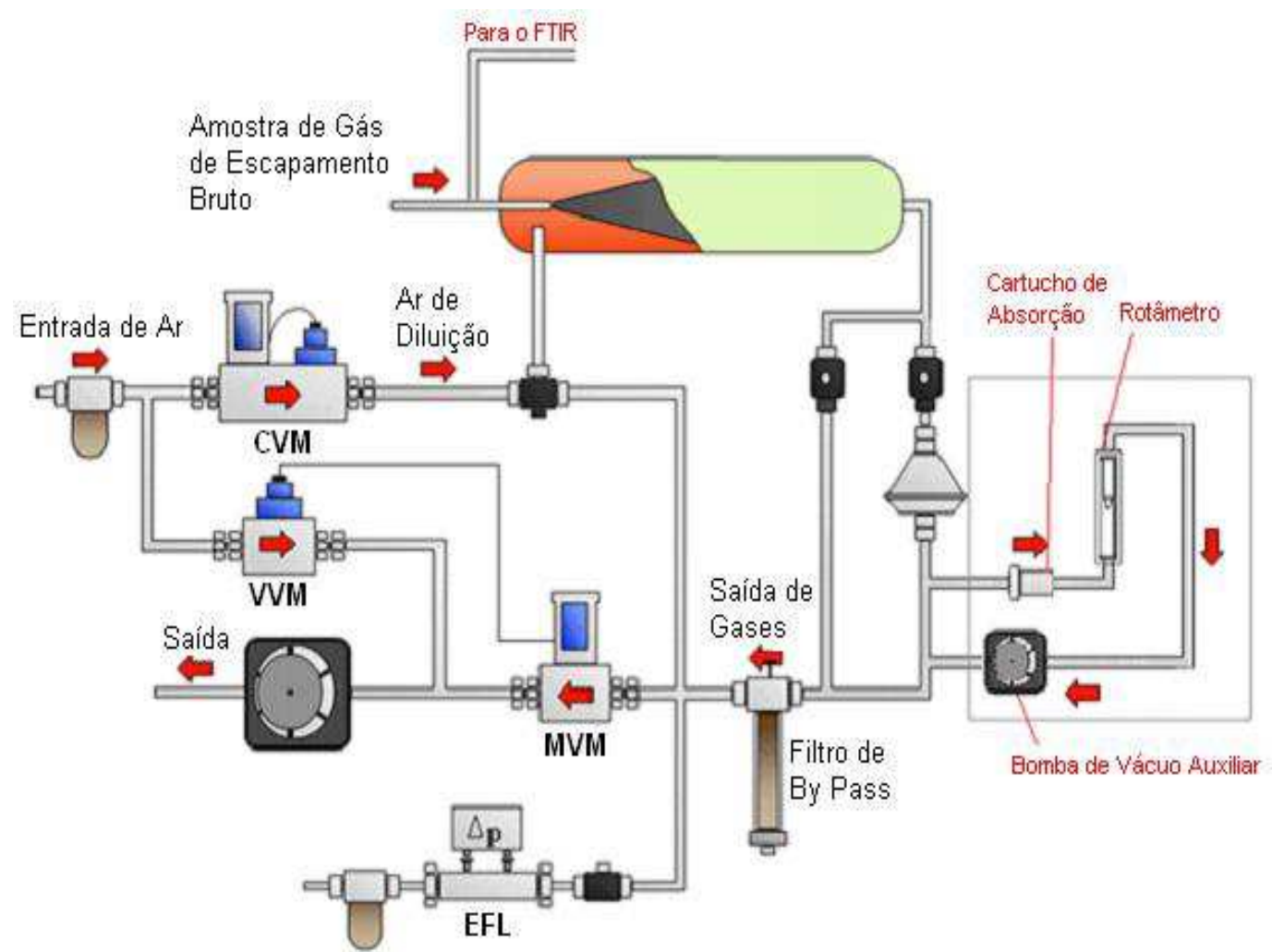

Figura 5. Esquema de ligação da amostragem de aldeídos no amostrador de material particulado.

Onde, MFC é o controlador de vazão mássica, MFV é a válvula de vazão mássica, MFM é o medidor de vazão mássica e LFE é o elemento de fluxo laminar.

Com base no esquema apresentado na Figura 5 foram feitas diversas modificações no equipamento de amostragem de material particulado do laboratório. As modificações incluíram o uso de uma bomba de amostragem adicional de forma a não prejudicar a amostragem de material particulado feita pelo equipamento.

Na figura 6 é apresentada uma foto do equipamento de amostragem de material particulado (SMART SAMPLER) com as modificações implantadas e a indicação da localização do cartucho, medidor de vazão e sistema de by-pass. 


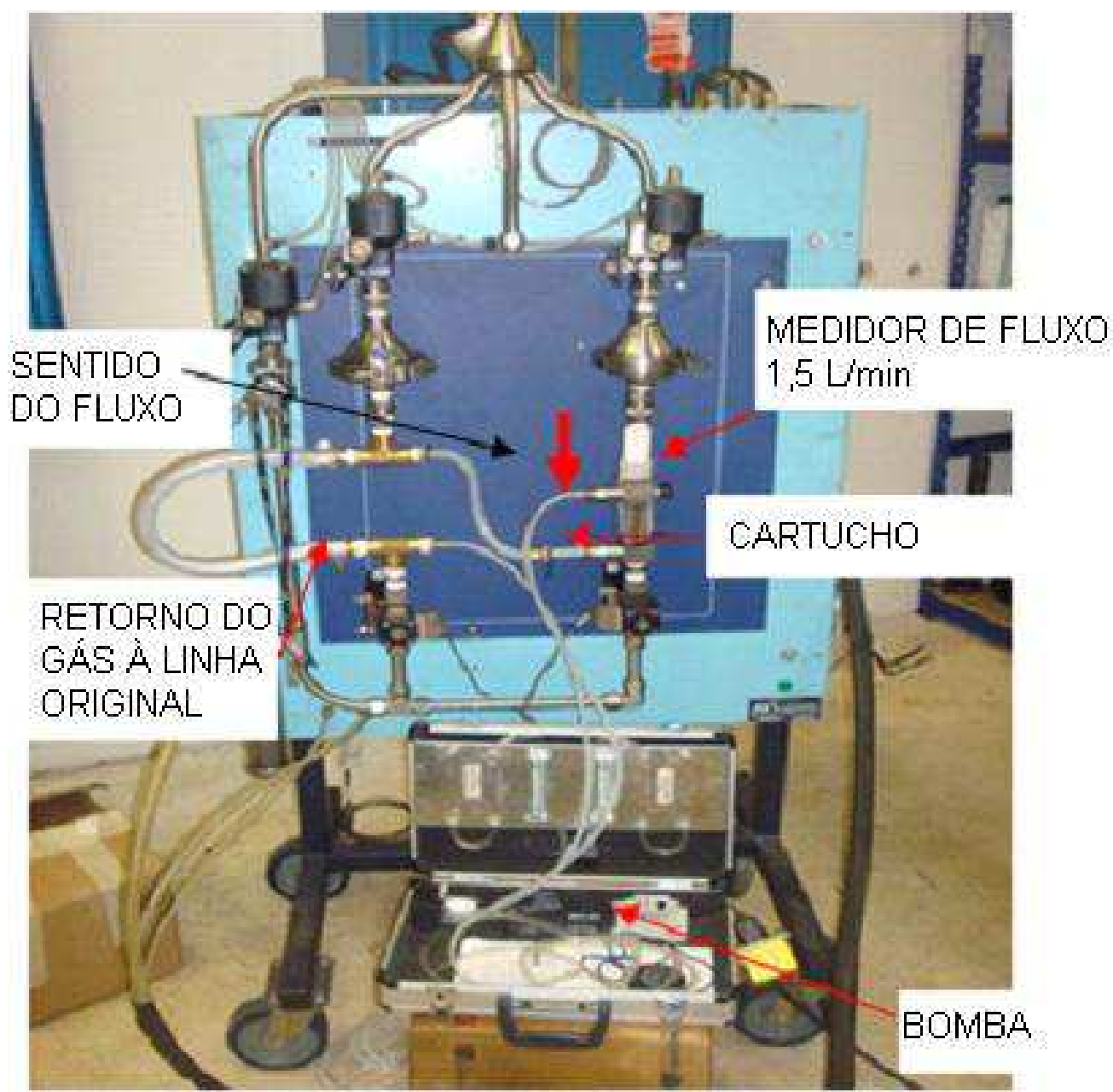

Figura 6. Foto do equipamento amostrador de material particulado com as modificações para a amostragem de aldeídos

Foi usado um mesmo cartucho com DNPH para todo o ensaio de emissões do tipo ESC. Diversos procedimentos analíticos foram desenvolvidos no LACTEC para a retirada e manuseio da amostra do cartucho de forma precisa.

\section{RESULTADOS DE EMISSÕES DE ALDEÍDOS EM VEÍCULOS}

Para entender como são formados os aldeídos em um veículo a diesel, sem sistema de catalisador, foram feitos ensaios usando o ciclo da norma NBR 6601. O ensaio incluiu uma partida a frio (fase 1 - $505 \mathrm{~s}$ ), e também o ciclo da fase quente (fase 3 - 505s).

Foi usado o sistema FTIR para a medição dos aldeídos, sendo que a figura 7 apresenta os resultados do acetaldeído e a figura 8 os resultados do formaldeído.

Valores mais elevados de aldeídos ocorreram nos primeiros 200 segundos do ciclo, fase fria. Pode ser verificado que na fase que 3 (partida a quente), houve grande redução da formação dos aldeídos. O formaldeído também é maior que o acetaldeído, sendo uma característica dos motores diesel. 


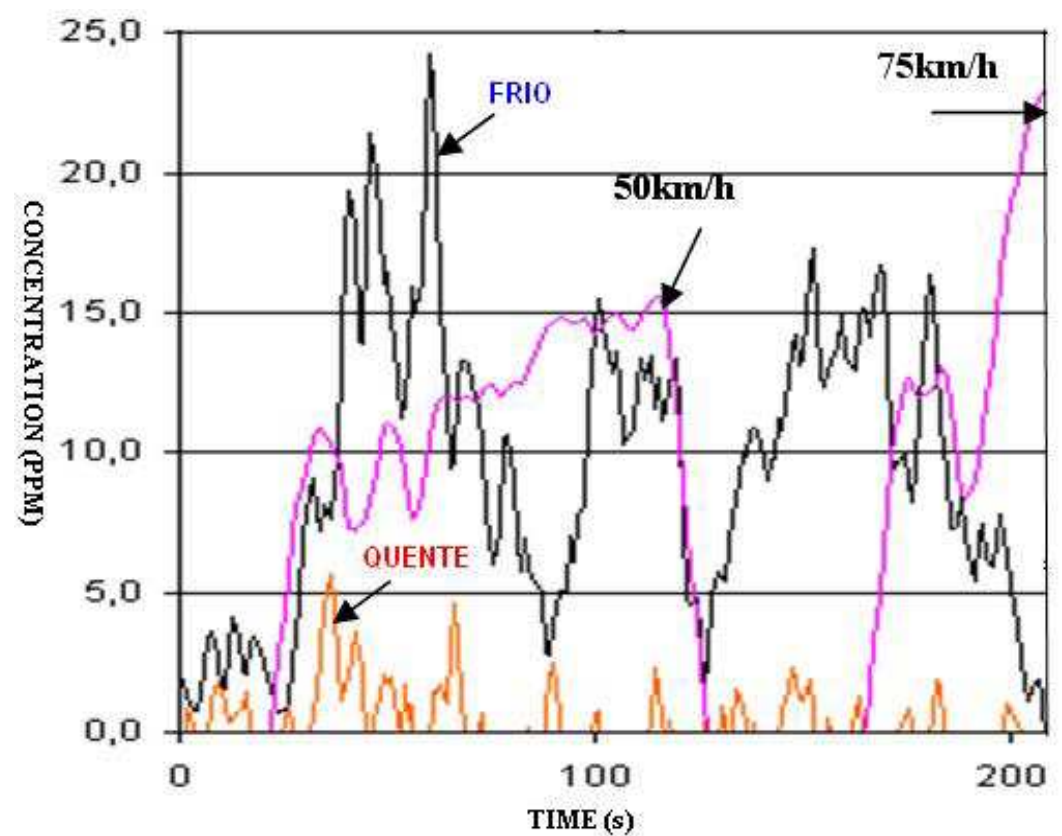

Figura 7. Acetaldeído na fase 1 (partida a frio) e fase 3 (partida a quente) - V3.

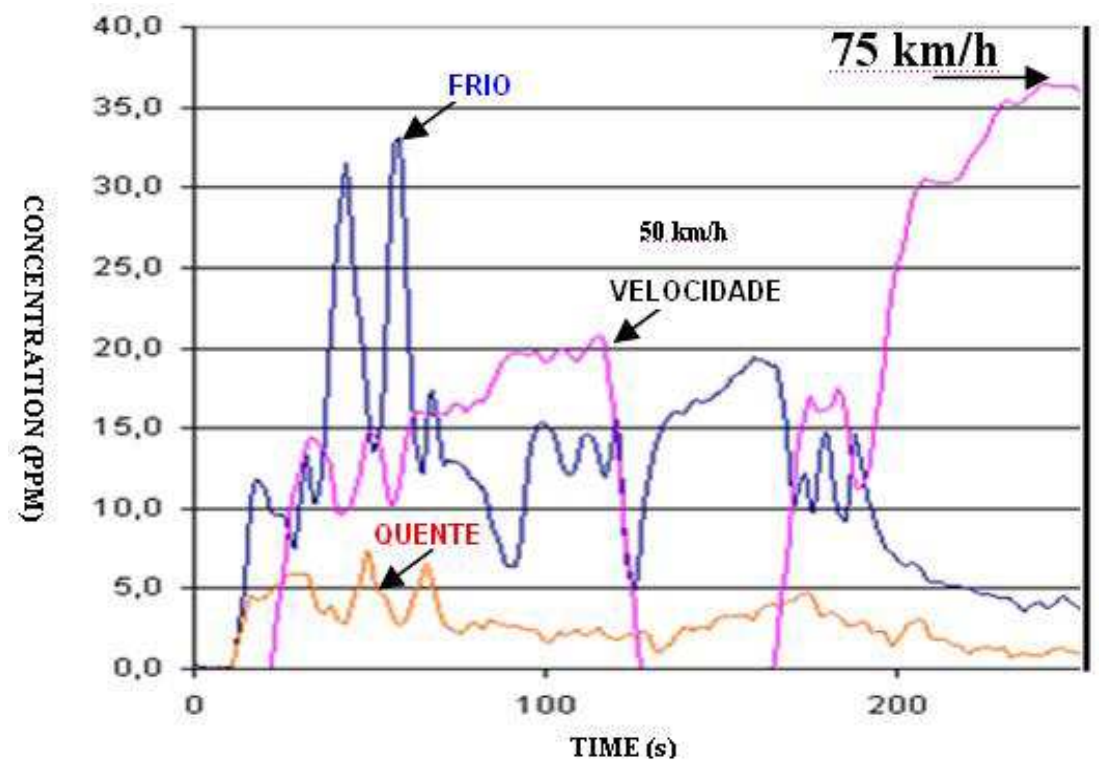

Figura 8. Formaldeído na fase 1 (partida a frio) e fase 3 (partida a quente) - V3.

Os veículos do tipo SUV e picape, da fase L3 do PROCONVE (1997-2006), usando gasolina ou etanol possuem um limite de emissões de aldeídos totais (formaldeído e acetaldeído) de $60 \mathrm{mg} / \mathrm{km}$, valor que será usado como referência máxima do eixo Y nos gráficos a seguir (figura 9).

Foram feitas análises estatísticas (ANOVA e FISHER LSD) para o formaldeído, acetaldeído e aldeídos totais (formaldeído + acetaldeído).

Os gráficos das médias dos aldeídos totais (formaldeído + acetaldeído) são apresentados a seguir, sendo que no eixo Y foi escolhido o valor máximo de $60 \mathrm{mg} / \mathrm{km}$, que corresponde ao limite do PROCONVE para veículos movidos a gasolina e/ou etanol de modelos do tipo picape e/ou SUV. 
Com relação ao formaldeído e acetaldeído, conforme mencionado anteriormente eles se encontram no escapamento do veículo diesel na ordem de grandeza de $70 \%$ para o formaldeído e $30 \%$ para o acetaldeído.

Na figura 9 é apresentada a média da emissão de aldeídos totais, para pelo menos 3 ensaios, dos veículos testados em função do teor e tipo de biodiesel. As barras indicam o intervalo de confiança dos valores para $95 \%$ de confiança..
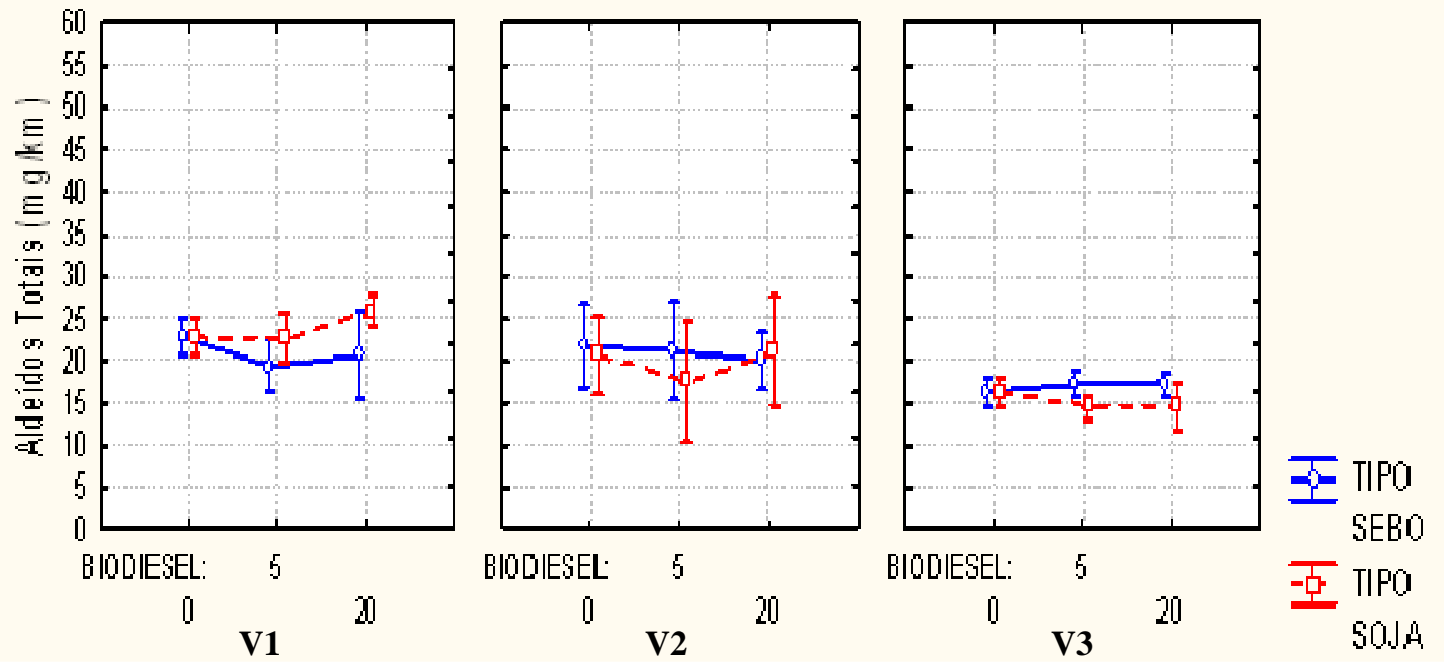

Figura 9. Emissão de Aldeídos Totais dos veículos em função do teor e tipo de biodiesel.

Na Tabela 3 são apresentados os resultados da análise estatística (método ANOVA FISHER LSD) das médias de aldeídos totais, sendo colocado o símbolo "=" para sinalizar quando não há diferença significativa, "AUM" para quando há aumento de emissões e "DIM" quando ocorre diminuição do valor em relação ao combustível de referência diesel S50 (coluna da esquerda da tabela). 
Tabela 3. Resultados da análise estatística das médias de Aldeídos Totais para os veículos (V1,V2,V3)

\begin{tabular}{|c|c|c|c|c|c|}
\hline \multicolumn{6}{|c|}{ ALDEIDOS TOTAIS } \\
\hline \multirow{3}{*}{\multicolumn{2}{|c|}{ REFERËNCIA }} & \multicolumn{4}{|c|}{$V_{1}$} \\
\hline & & \multicolumn{2}{|c|}{ SOJA } & \multicolumn{2}{|c|}{ SEBO } \\
\hline & & B5 & B20 & B5 & B20 \\
\hline & $\mathbf{0}$ & $=$ & $=$ & $=$ & $=$ \\
\hline \multirow{2}{*}{ SOJA } & B5 & & AUM & $=$ & \\
\hline & $\mathrm{B} 20$ & & & & $\bar{D} \mid \mathrm{M}$ \\
\hline \multirow[t]{5}{*}{ SEBO } & B5 & & & & $=$ \\
\hline & & \multicolumn{4}{|c|}{$\sqrt{2}$} \\
\hline & & \multicolumn{2}{|c|}{ SOJA } & \multicolumn{2}{|c|}{ SEBO } \\
\hline & & B5 & B20 & B5 & B20 \\
\hline & 0 & $=$ & $=$ & $=$ & $=$ \\
\hline \multirow{2}{*}{ SOJA } & B5 & & $=$ & $=$ & \\
\hline & $\mathrm{B} 20$ & & & & $=$ \\
\hline \multirow[t]{5}{*}{ SEBO } & B5 & & & & $=$ \\
\hline & & \multicolumn{4}{|c|}{$\sqrt{3}$} \\
\hline & & \multicolumn{2}{|c|}{ SOJA } & \multicolumn{2}{|c|}{ SEBO } \\
\hline & & B5 & B2U & B5 & $\mathrm{B} 2 \mathrm{~T}$ \\
\hline & 0 & $=$ & $=$ & $=$ & $=$ \\
\hline \multirow{2}{*}{ SOJA } & B5 & & $=$ & $=$ & \\
\hline & $\mathrm{B} 20$ & & & & $=$ \\
\hline SEBO & B5 & & & & $=$ \\
\hline
\end{tabular}

Com base na figura 9 e na tabela 4, pode ser verificado que:

- Os veículos V2 e V3 não apresentaram diferenças significativas da emissão de aldeídos para todos os teores de biodiesel de soja e sebo testados. Os veículos também não foram sensíveis ao tipo de biodiesel utilizado.

- O veículo V1 apresentou tendência de aumento dos aldeídos com o uso de B20 em relação ao B5 de soja. Esse veículo também apresentou valores maiores de emissões com o uso de B20 de soja em comparação ao B20 de sebo.

O veículo V1 foi o único sensível ao tipo de biodiesel usado, o que pode ser explicado pelo fato dele ser o único veículo que possui sistema de injeção e controle mecânico e também por não estar equipado com dispositivo eletrônico de partida a frio com vela de pré-aquecimento. Como mencionado anteriormente, a maior parte da formação dos aldeídos é na fase fria do ciclo de ensaio, ou seja, nos primeiros 200 segundos.

Em resumo, os valores de aldeídos totais encontrados nos veículos diesel testados estão na faixa de 15 a $25 \mathrm{mg} / \mathrm{km}$, portanto abaixo do limite de $60 \mathrm{mg} / \mathrm{km}$ da fase L-3 de veículos comerciais leves com peso acima de $1700 \mathrm{~kg}$ para um modelo a gasolina ou etanol equivalente. A adição de biodiesel nos teores de B5 e B20 não provocou variações significativas que comprometam seu uso, considerando os resultados obtidos nos veículos ensaiados.

Por se tratar de uma técnica nova em implantação no país, ainda existem pontos de melhoria, principalmente no sentido de se reduzir a dispersão de resultados para uma melhor comparação da influência de diferentes tipos de combustíveis. 


\section{ENSAIOS DE EMISSÕES DE ALDEÍDOS EM MOTORES}

Todos os ensaios de emissões em motor foram feitos segundo a norma NBR 15634, seguindo o ciclo ESC por se tratar de motores com tecnologia EURO III.

Para as emissões legisladas e de aldeídos, foram feitos ensaios no LACTEC em motor com tecnologia EURO III. Conforme mencionado anteriormente para as emissões de aldeídos, foram feitos ensaios com o uso da metodologia desenvolvida por cromatografia e também pela técnica de FTIR.

Foi necessária a elaboração de uma metodologia inédita no país para a amostragem dos aldeídos em banco de provas de motor. Foi feita uma modificação no equipamento amostrador de material particulado existente, que permitisse a instalação de cartuchos de sílica impregnados com 2,4-DNPH para a amostragem de aldeídos. A sequência de ensaios de motor teve o diesel S50 (B0) como referência e depois foi feita a adição de biodiesel nos teores de B5 e B20. Antes de cada nova série de ensaios foi feita a troca do filtro do combustível do motor. Na tabela 4 são apresentados os resultados dos poluentes medidos CO, NMHC, NOx, MP e dos aldeídos.

Tabela 4. Resultados dos aldeídos totais por cromatografia líquida.

\begin{tabular}{|c|c|c|}
\hline \multicolumn{2}{|c|}{ BIODIESEL } & NÃO LEGISLADOS \\
\cline { 3 - 3 } & ALDEÍDOS TOTAIS (mg/kWh) \\
\hline \multirow{3}{*}{ SOJA } & 0 & 2,93 \\
\cline { 2 - 3 } & B5 & 2,40 \\
\cline { 2 - 3 } & B20 & 2,83 \\
\hline \multirow{3}{*}{ SEBO } & 0 & 2,93 \\
\cline { 2 - 3 } & B5 & 3,43 \\
\cline { 2 - 3 } & B20 & 3,55 \\
\hline
\end{tabular}

$\mathrm{Na}$ tabela 5 são apresentados os resultados da análise estatística (FISHER LSD) para os aldeídos medidos por cromatografia e por FTIR. Pode ser observado dessa tabela, que ambas as técnicas de medição (cromatografia e FTIR) apresentaram a mesma tendência em função do aumento de teor de biodiesel.

Tabela 5. Resultados da análise estatística das médias de aldeídos totais em Motor EURO III

\begin{tabular}{l|c|c|c|c|c}
\hline \multicolumn{2}{c|}{ MOTOR } & \multicolumn{4}{c}{ ALDEIDOS TOTAIS } \\
\hline \multirow{2}{*}{ REFERÊNCIA } & \multicolumn{4}{|c}{ SOJA } \\
\cline { 3 - 6 } & CROMATOGRAFIA & \multicolumn{2}{c}{ FTIR } \\
\cline { 3 - 6 } & B5 & B20 & B5 & B20 \\
\hline \multirow{2}{*}{ SOJA } & 0 & DIM & $=$ & DIM & $=$ \\
\cline { 3 - 6 } & B5 & & AUM & AUM \\
\hline \multirow{2}{*}{} & \multicolumn{4}{|c}{ SEBO } \\
\cline { 3 - 6 } & CROMATOGRAFIA & FTIR \\
\cline { 3 - 6 } & B5 & B20 & B5 & B20 \\
\hline \multirow{2}{*}{ SEBO } & 0 & AUM & AUM & AUM & AUM \\
\cline { 2 - 6 } & B5 & & $=$ & & $=$ \\
\hline
\end{tabular}


Com base nas tabelas 4 e 5 pode ser comentado que:

- Para o biodiesel de sebo houve uma tendência de aumento das emissões para o uso de B5 e de B20, quando comparado ao diesel S50. A comparação B5 x B20 não foi significativa.

- Para o biodiesel de soja com o uso da mistura B5 foram obtidos no experimento valores menores do que o diesel S50 e do que o da mistura B20, porém essas diferenças apesar de estatisticamente significativas são da ordem de $0,5 \mathrm{mg} / \mathrm{kWh}$ valor próximo ao limite do método.

Como não existe legislação internacional para aldeídos em motores pesados do ciclo diesel, não estão disponíveis na literatura valores típicos de emissão de aldeídos em $\mathrm{mg} / \mathrm{kWh}$ do ensaio ESC para que sejam comparados com os valores obtidos nos ensaios desse projeto. Porém cabe registrar que todos os valores encontrados foram baixos e menores do que $4 \mathrm{mg} /$ kWh para todos os combustíveis testados.

\section{CONSIDERAÇÕES FINAIS E CONCLUSÃO}

As emissões de aldeídos para os veículos comerciais leves (SUV e picape) foram da ordem de $20 \mathrm{mg} / \mathrm{km}$, abaixo do limite de $60 \mathrm{mg} / \mathrm{km}$ para os veículos a álcool e a gasolina de categoria equivalente. A adição de biodiesel até $20 \%$ (v/v) não aumentou significativamente os aldeídos independente do tipo de biodiesel usado (soja ou sebo). A excessão foi o veículo V1, que foi sensível ao tipo de biodiesel e ao percentual usado na mistura, devido ao fato de possuir uma tecnologia mais antiga, com sistema de injeção de combustível mecânico e sem sistema de pré-aquecimento.

Para os ensaios em motor EURO III, houve uma tendência de aumento das emissões de aldeídos com o uso de B5 e B20 de Sebo, em comparação ao diesel S50. Para o biodiesel de Soja, não houve diferença significativa na comparação do B20 com o S50, porém houve tendência de redução com o uso da mistura B5.

Com base nos resultados encontrados para os veículos e motor ensaiados, foi verificado que até o percentual de $20 \%$ de biodiesel na mistura, não são esperados aumentos significativos de emissões de aldeídos que prejudiquem o uso dessa mistura. Como a emissão de aldeídos é muito reduzida com o uso de catalisadores de oxidação, e esses dispositivos estão sendo implantados nos novos veículos diesel da fase L6 e P7, é esperada uma redução significativa desse tipo de poluente. 


\section{REFERÊNCIAS}

[1] ASSOCIAÇÃO BRASILEIRA DE NORMAS TÉCNICAS- ABNT, NBR 12026, March/2002 - Light Duty Vehicles - Determination of Aldehydes and Ketones in Exhaust Gas by Liquid Chromatography- DNPH Method.

[2] ABRANTES, R., ASSUNÇÃO, J. V., HIRAI, E. Y. Caracterização das Emissões de Aldeídos de Veículos do Ciclo Diesel. Rev Saúde Pública 2005; 39(3): 479-85.

[3] ASSOCIAÇÃO BRASILEIRA DE NORMAS TÉCNICAS - ABNT - NBR 6601/2005 Road Vehicles Determinations of Hydrocarbon (HC), Carbon Monoxide (CO), Nitrogen Oxides (NOx), Carbon Dioxide (CO2) and Particulate Material on Exhaust Gas.

[4] CAPLAIN, ET AL. Emissions of Unregulated Pollutants from European Gasoline and Diesel Passenger Cars. Science Direct, 2006.

[5] SHIOTANI, H., GOTO, S., KINOSHITA, K., NIKOLIC, D. Characteristics of Aldehydes and VOCs Emission from Off-road Engines. SAE 2006-32-0023. Small Engine Technology Conference and Exhibition, San Antonio, Texas, USA, November, 2006.

[6] ARAPAKI, et al. Regulated and Unregulated Emissions Characteristics of a Diesel Vehicle Operating with Diesel/Biodiesel Blends. SAE Technical Paper, 2007-01-0071.

[7] PENG, C., ET AL. Effects of the Biodiesel Blend Fuel on Aldehyde Emissions from Diesel Engine Exhaust. Science Direct, Atmospheric Environment, 2008.

[8] PI-QIANG TAN *, ZHI-YUAN HU, DI-MING LOU. Regulated and Unregulated Emissions from a Light-Duty Diesel Engine with Different Sulfur Content Fuels. Elsevier, Fuel 88 (2009) 1086-1091.

[9] KARAVALAKIS, G., ALVANOU F., STOURNAS S., BAKEAS E. Regulated and Unregulated Emissions of a Light Duty Vehicle Operated on Diesel/Palm-Based Methyl Ester Blends Over NEDC and a Non-Legislated Driving Cycle 13 b. Elsevier, Fuel 88 (2009) 10781085 .

[10] GUARIEIRO, L. L. N.; DE SOUZA, A. F.; TORRES, E. A.; DE ANDRADE, J. B. Emission Profile of 18 Carbonyl Compounds, CO, CO2, and NOx Emitted by a Diesel Engine Fuelled with Diesel and Ternary Blends Containing Diesel, Ethanol and Biodiesel or Vegetable Oils. Elsevier, Atmospheric Environment 43 (2009) 2754-2761.

[11] SHAH, A. N., YUN-SHAN, G., JIAN-WEI,T.. Carbonyls Emission Comparison of a turbocharged Diesel Engine fuelled with Diesel, Biodiesel, and Biodiesel-Diesel blend. Jordan Journal of Mechanical and Industrial Engineering (JJMIE). Volume 3 , Number 2, pages 111118, June 2009.

[12] HE, C., GE Y., SHAH, A. et al., Comparison of carbonyl compounds emissions from diesel engine fueled with biodiesel an diesel. Atmospheric Environment, 2 April 2009.

[13] STOREY, J., THOMAS, J.F., LEWIS, S.A., et al. , Particulate Matter and Aldehyde Emissions from idling Heavy-Duty Diesel Trucks. SAE 2003-01-0289. SAE 2003 WORLD CONGRESS, DETROIT, USA.

[14] KARAVALAKIS G, BAKEAS E, STOURNAS S. Influence of oxidized biodiesel blends on regulated and unregulated emissions from a diesel passenger car. Environ Sci Technol. 2010 Jul 1;44(13):5306-12. doi: 10.1021/es100831j.2010.

[15] ANDERSON, L.,G. Effects of Biodiesel Fuels Use on Vehicle Emissions. Journal of Sustainable Energy \& Environment3 (2012) 35-47, 2012.

[16] JO-YU CHIN, STUART A. BATTERMAN, WILLIAM F. NORTHROP, STANISLAV V. BOHAC, AND DENNIS N. ASSANIS. Gaseous and Particulate Emissions from Diesel Engines at Idle and under Load: Comparison of Biodiesel Blend and Ultralow Sulfur Diesel Fuels. Energy \& Fuels doi: 10.1021/ef300421h. 2012 